\title{
Estudo Comparativo do Modelo de Atenção Farmacêutica entre Brasil e Canadá
}

\author{
Caroline da Rocha Junqueira, ${ }^{1}$ Amanda Felipe Portella, ${ }^{2}$ \\ Viviane Cecilia Kessler Nunes Deuschle, ${ }^{3}$ Josiane Woutheres Bortolotto , ${ }^{4}$ \\ Gabriela Bonfanti Azzolin ${ }^{5}$
}

\begin{abstract}
RESUMO
Objetivo: Avaliar a metodologia de atenção farmacêutica canadense e comparar com a metodologia brasileira, visando ao aperfeiçoamento da atenção farmacêutica no Brasil. Métodos: Foram utilizados artigos já publicados disponíveis no SciELO, Elsevier, Google Scholar, Medline-Pubmed, bem como relatórios e materiais disponíveis nos sites dos órgãos reguladores da profissão farmacêutica de ambos os países. Resultados e discussão: O profissional farmacêutico brasileiro, quando comparado ao profissional canadense, não encontra uma legislação precisa que o oriente quanto à execução das atividades de atenção farmacêutica, não possui uma metodologia específica ou ainda uma estratégia de ação para a aplicação dessa atividade, e também carece de educação de qualidade para a execução da atenção farmacêutica. Conclusão: Para que ocorra um meIhoramento na atenção farmacêutica no Brasil é necessária a criação de uma legislação fortificada e metodologias de atenção farmacêutica voltadas à realidade do país.
\end{abstract}

Palavras-chave: Atenção farmacêutica. Assistência farmacêutica. Farmácia. Medicamentos.

\section{COMPARATIVE STUDY OF PHARMACEUTICAL CARE MODELS: BRAZIL VS CANADA}

\section{ABSTRACT}

Objective: To evaluate the Canadian pharmaceutical care methodology, and compare with the Brazilian methodology, in order to point out the needs of improvement of pharmaceutical care in Brazil. Methods: The articles used were published in SciELO, Elsevier, Google Scholar, Medline, Pubmed, as well as reports and information available on sites of the regulatory authority of the pharmacist profession in both countries. Results and discussion: Brazilian pharmacists, when compared to Canadian professionals, did not have a specific methodology, legislation or strategy in the pharmaceutical care. It still faces some difficulties in the professional education of the pharmacist that will carry out this service. Conclusions: It is necessary to create a fortified legislation and methodologies to attend the needs of Brazil in order to improve its pharmaceutical care field. Keywords: Pharmaceutical care. Pharmaceutical attendance. Pharmacy. Medicine. 


\section{INTRODUÇÃO}

Atenção farmacêutica é uma prática designada ao profissional farmacêutico que assegura que a conduta medicamentosa seja adequada. Assim, tem a finalidade de assegurar e promover a saúde; monitorar e prevenir doenças; modificar medicações e promover segurança e eficácia no tratamento em busca de um resultado positivo para o paciente (AMERICAN..., 2015).

A atuação do profissional farmacêutico na comercialização de medicamentos e prestação de serviços aos pacientes vem sendo discutida e repensada desde 1960, quando já se observava a necessidade da aproximação entre o profissional e o paciente (PEREIRA; FREITAS, 2008). A expressão "Atenção Farmacêutica" foi utilizada pela primeira vez em 1990 por Hepler e Strand, que a descreveram como um instrumento para buscar resultados satisfatórios para os pacientes em tratamento medicamentoso. Essa metodologia de promoção de saúde é relativamente nova e vem aos poucos conquistando o seu espaço no território acadêmico (MORAK et al., 2010; ARAÚJO; UETA; FREITAS, 2005).

O Canadá possui como característica de suas farmácias a aplicação de inúmeros recursos para que haja a atenção e assistência ao paciente, incluindo análises de medicamentos, gerenciamento de doenças crônicas, serviços de imunização e programas de bem-estar (CANADIAN..., 2015). A utilização de diferentes planos de saúde e documentação para pesquisar e examinar a medicação utilizada pelo paciente permite que o tratamento seja otimizado, trazendo menor impacto negativo ao paciente, além de diminuir erros de administração e prescrição (AMIR, 2011). O país ainda conta com o auxílio governamental para que haja revisão nas prescrições e assistência farmacêutica de forma mais rígida, e ainda parte dos Estados canadenses conta com programa governamental de remuneração por prescrição revisada desde o ano de 2007 (CANADA, 2015). Além deste, destacam-se os demais programas que se diferem na sua metodologia, porém possuem o mesmo objetivo de trazer meIhoramentos à saúde do paciente, identificar possíveis problemas relacionados com os medicamentos e monitorar o progresso do indivíduo em sua terapia medicamentosa (PAMMETT; JORGENSON, 2014).

No Brasil, o acompanhamento do tratamento medicamentoso como forma de prevenir e promover a saúde ainda está em crescimento. $O$ surgimento do conceito de atenção farmacêutica como forma de promoção da saúde no país ainda é muito recente. No ano de 2002, o Consenso Brasileiro de Atenção Farmacêutica foi criado pela Organização Pan-Americana da Saúde para estabelecer a forma de atuação do farmacêutico, delimitando seus deveres para com a sociedade (OPAS, 2002). Como muitos países em desenvolvimento, porém, o Brasil possui um conceito cultural sobre farmácia, constituindo este um estabelecimento caracterizado somente pela comercialização de medicamentos, poucos são os estabelecimentos que promovem a atenção farmacêutica no país. A falta de reconhecimento do farmacêutico como provedor de educação em saúde, a escassez de infraestrutura e apoio dos estabelecimentos são obstáculos que afastam o profissional da prática de atenção (FARINA; ROMANO-LIEBER, 2009).

Sabendo, portanto, das dificuldades encontradas na área da atenção farmacêutica no Brasil, este trabalho tem por objetivo pesquisar as metodologias de atenção farmacêutica canadenses e compará-las ao modelo brasileiro. Também visa a analisar as leis regentes da profissão farmacêutica do Canadá e do Brasil e avaliar a base curricular da educação recebida pelos estudantes de Farmácia e seu preparo como profissional farmacêutico. Ademais, esta pesquisa irá comparar os parâmetros entre os dois países em busca de exemplos de inovação e melhoramento da atenção farmacêutica em farmácias brasileiras.

\section{METODOLOGIA}

A elaboração deste artigo foi realizada por meio de uma revisão bibliográfica narrativa. Foram usados como base de literatura para a estruturação deste trabalho artigos publicados nas bases de dados disponíveis no SciELO, Elsevier, Google Scholar, Medline-Pubmed. Também foram analisados parâmetros brasileiros e canadenses referente a leis e educação superior disponíveis nas páginas eletrônicas dos governos brasileiro e canadense, bem como os órgãos reguladores da profissão farmacêutica de ambos os países. Não houve limitação quanto às datas dos artigos pesquisados e o critério de inclusão dos artigos foi realizado de acordo com a relevância do assunto para a proposta desta revisão, em língua portuguesa e inglesa. Foram excluídos dissertações, teses e resumos.

\section{RESULTADOS E DISCUSSÃO}

\section{Sistema de Saúde e Atenção Farmacêutica}

O reconhecimento da Atenção Farmacêutica foi moldado a partir de estudo realizado por Hepler (1987), no qual foram evidenciadas as responsabilida- 
des do profissional farmacêutico sobre as medicações devido ao seu profundo conhecimento das drogas e terapias. Mais tarde, Hepler e Strand (1990) formularam o conceito de Atenção Farmacêutica, que é definido como "a provisão responsável do tratamento farmacológico com o objetivo de alcançar resultados satisfatórios na saúde, melhorando a qualidade de vida do paciente". Esse conceito foi então aceito pela Organização Mundial da Saúde (OMS) em 1994, que posteriormente caracterizou detalhadamente a função do profissional farmacêutico dentro da Atenção Farmacêutica, delimitando seus deveres e responsabilidades para com a saúde do paciente.

Antes mesmo de a Atenção Farmacêutica ganhar importância mundial, o Canadá já havia reconhecido a magnitude das ações do farmacêutico diante da sociedade. Em 1970 o país já discutia assuntos pertinentes à escolha de terapias medicamentosas, chamada de "Opinião Farmacêutica", em que o farmacêutico já tinha liberdade para discutir importantes aspectos das drogas escolhidas. Atualmente o farmacêutico tem o papel de promover resultados positivos à saúde do paciente, melhorar e otimizar a terapia medicamentosa, garantindo que os benefícios estejam sendo utilizados de forma adequada, e por fim, reduzir o uso inadequado de drogas e seu desperdício (CANADA, 2015; CANADIAN..., 2015; PEREIRA; FREITAS, 2008).

No Brasil, a Atenção Farmacêutica começou a ser discutida oficialmente após o reconhecimento mundial da prática. Em 2002, o Consenso Brasileiro de Atenção Farmacêutica foi criado com o objetivo de definir a Atenção Farmacêutica como política de saúde, no entanto ainda não existe nenhum projeto para adesão da Atenção Farmacêutica em estabelecimentos comerciais. Em estudo realizado por Ambiel e Mastroianni (2013), são ressaltadas as dificuldades para a instituição da Atenção Farmacêutica, como a carga de trabalho excessiva, a falta de infraestrutura física para atendimento privativo e carência na formação profissional (CONSENSO..., 2002).

Acima de tudo, destaca-se o perfil evolutivo de ambos os países como o principal fator para a contribuição da realização da atenção farmacêutica. O Canadá é um país desenvolvido que possui um alto índice de renda per capita e economia industrial avançada, seu índice de desenvolvimento humano é de 0,911 (PNDU, 2013). O país possui uma administração central, porém cada província é responsável pelos seus respectivos programas sociais e administração da saúde da sua população. Também é considerado um país com experiência e efetividade no setor da Atenção Farmacêutica (MARCHILDON, 2013). O Brasil, por outro lado, é um país em desenvolvimento e seu índice de desenvolvimento humano é de 0,730 . Possui similaridades com o Canadá quanto a sua organização, dispõe de um sistema político federativo constituído por três esferas de governo - União, Estados e municípios - e cada uma destas esferas possui independência administrativa (SOUZA, 2002).

A história e percurso da instituição do sistema de saúde também difere entre os países. O Canadá teve o primeiro planejamento de sistema de saúde universal para a população em 1947, na província de Saskatchewan. Com o passar dos anos, esta província aprimorou o seu atendimento à população, oferecendo um serviço de saúde mais amplo. Em 1957 o Parlamento Canadense delineou as condições que cada província deveria atingir para poder obter o repasse dos recursos financeiros destinados à saúde. Em 1971 todas as províncias passaram a receber cobertura nos atendimentos hospitalares e serviços médicos. Mais tarde esses serviços foram especificados na legislação e ficou conhecido como Canada Health Act, sendo devidamente regulamentado em 1984 . Nessa legislação foram estabelecidos os critérios e condições dos serviços de saúde prestados sob a lei provincial, ou seja, administração pública, integralidade, universalidade, portabilidade e acessibilidade (MARCHILDON, 2013; CANADA, 2015).

Em 1953 foi criado no Brasil o Ministério da Saúde, que na época era responsável somente por ações de controle de endemias e prevenção de doenças. Esses atendimentos eram de caráter universal, ou seja, toda a população poderia ter acesso. A assistência ocorria em hospitais específicos em cada região, e existia certa problemática para que toda a população fosse atendida. A criação do Sistema Único de Saúde (SUS), em 1988, trouxe os princípios de integralidade e igualdade de acesso à saúde e um dos principais objetivos de sua criação era a execução de ações de assistência terapêutica integral, inclusive farmacêutica. Essa participação direta do farmacêutico dentro do SUS trouxe liberdade para que o profissional participasse do acompanhamento da conduta medicamentosa dos pacientes, retomando o seu papel no âmbito da saúde. O Encontro Nacional de Assistência Farmacêutica e a Política de Medicamentos em 1988 foram também importantes como formação de estratégias para a criação de ações na área da saúde, mas somente em 2002 foi criado o Consenso Brasileiro de Atenção Farmacêutica que abordou o assunto Atenção Farmacêutica como política de saúde (FIOCRUZ, 2015; PEREIRA; FREITAS, 2008; SOUZA, 2002; BRASIL, 1990). 
Então, os farmacêuticos brasileiros obtiveram uma grande conquista, a Resolução no 586, de 29 de agosto de 2013, elaborada pelo Conselho Federal de Farmácia, que regula o farmacêutico como profissional habilitado para recomendar medicamentos aos pacientes, ou seja, o farmacêutico é apto para prescrever medicamentos e outros produtos com finalidade terapêutica de venda livre ou medicamentos cuja dispensação exija prescrição médica, desde que haja um diagnóstico médico prévio de tal enfermidade (CONSELHO..., 2013b). De maneira geral, entende-se como prescrição farmacêutica a ação de indicar algo ao paciente, entretanto, para mudar a visão da sociedade e de outros profissionais da saúde sobre o papel do farmacêutico é preciso que essas mudanças prosperem e que essas ações sejam de fato aplicadas (PEREIRA; FREITAS, 2008).

Diante destes fatos podemos especular que um dos principais fatores relacionados ao atraso da introdução da Atenção Farmacêutica no Brasil se dá devido à evolução lenta do país, como demonstrado na Figura 1. Além disso, a ação das políticas de saúde torna-se difícil em consequência da grande diversidade e desigualdade que existe no país. A diversidade entre a necessidade de saúde em uma população, diferentes tipos de ações e serviços necessários para servir à sociedade, a falta de capacitação pessoal e os recurso tecnológicos acabam, frequentemente, desestruturando o sistema de saúde e sua visão de direito da sociedade como um todo (SOUZA, 2002).

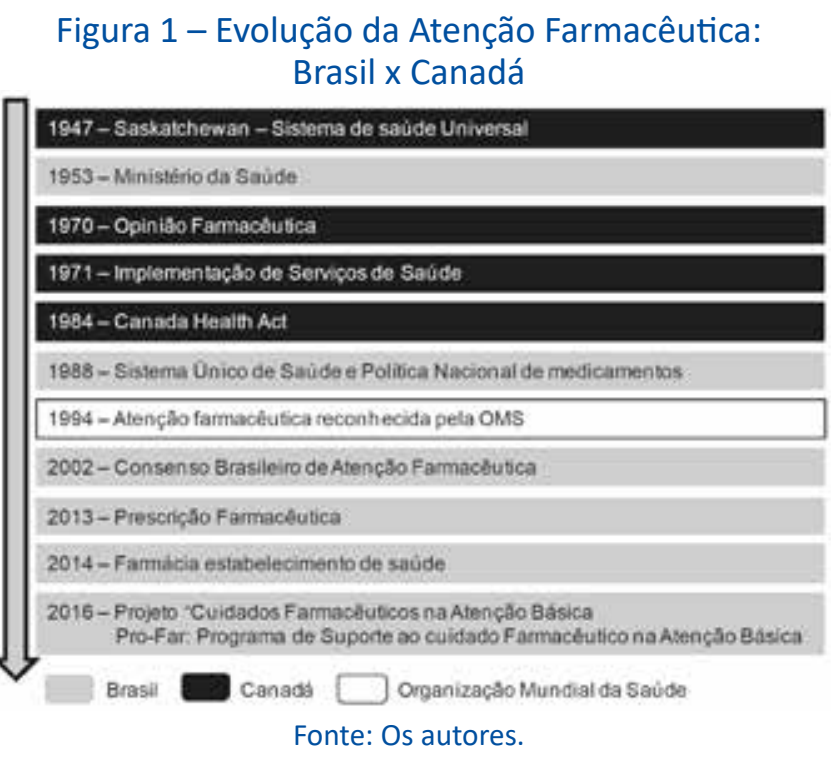

Além disso, são observadas diferenças na caracterização dos estabelecimentos. As farmácias comerciais canadenses são reconhecidas por oferecerem serviços de avaliação de medicamentos e gestão de doenças crônicas, como também serviços de vacina- ção e bem-estar. São chamadas de community pharmacies, e dentro do estabelecimento os pacientes encontram todas as informações necessárias sobre suas medicações. Os serviços nas community pharmacies estão disponíveis mediante consulta farmacêutica, gerenciamento e revisão dos medicamentos e monitoramento de testes de pressão arterial e glicose sanguínea direcionados para uma "comunidade" específica, ou seja, para um grupo-alvo determinado (CANADA, 2015; CANADIAN..., 2015; AMIR, 2011; FARRIS; FERNANDO FERNANDEZ-LLIMOS; BENRIMOJ, 2005).

Por outro lado, as farmácias brasileiras são caracterizadas pelo seu aspecto comercial, prevalecendo a distribuição de medicamentos industrializados. Com o avanço industrial as farmácias começaram a realizar somente a venda dos medicamentos produzidos por grandes laboratórios. Essa mudança acarretou a perda do contato direto do profissional farmacêutico com o paciente que existia durante o período pré-industrial. As ações realizadas pelos farmacêuticos nas farmácias comercias ocorrem somente durante a venda dos medicamentos, quando são sanadas as dúvidas sobre o seu uso (FILHO; BATISTA, 2011; PEREIRA; FREITAS, 2008). Com o intuito de mudar essa realidade, em 2014 foi promulgada a Lei no 13.021/2014, que transforma as farmácias privadas em estabelecimentos de saúde, possibilitando a atuação clínica do farmacêutico nesses locais (BRASIL, 2014). Quanto às instituições públicas de saúde, ainda se observa a falta de padronização nas tarefas realizada pelos farmacêuticos e um grande empenho em atividades administrativas, demonstrando, portanto, a ausência da realização de serviço farmacêutico especializado.

\section{Legislação em Atenção Farmacêutica}

A disposição das responsabilidades e aptidões do farmacêutico na legislação reafirma a importância do profissional na sociedade, assegurando sua capacidade para exercer a profissão. No Canadá as funções e requisitos básicos que cada profissional deve seguir para poder exercer a prática farmacêutica estão descritas no Model Standards of Practice for Canadian Pharmacists, desenvolvido pela The National Association of Pharmacy Regulatory Authorities (Napra). Em resumo, este documento informa que todo farmacêutico que exerça função em solo canadense poderá: revisar a prescrição médica, estender prescrições e ajustá-las quando necessário, interpretar testes laboratoriais, prescrever medicamento para condições menores e para quando não é preciso diagnóstico, 
além de substituir medicações em caso de baixa no estoque e administrar as medicações para demonstrar ao paciente o modo de uso (NAPRA, 2009).

Além disso, são delimitadas como funções do farmacêutico: organização e administração das medicações dispostas dentro da farmácia, responsabilidade no treinamento dos funcionários, assegurar que esteja ocorrendo a execução das leis vigentes e documentar suas decisões quando for aplicada a Atenção Farmacêutica. Cada província canadense, entretanto, possui uma legislação específica para as capacitações do profissional farmacêutico. Por exemplo, na província de Ontário o farmacêutico não pode realizar substituição terapêutica, enquanto na província de Alberta é permitido (NAPRA, 2012, 2009).

Quanto ao Brasil, a legislação que rege os farmacêuticos está disposta no Código de Ética Farmacêutica redigida pelo Conselho Federal de Farmácia (CFF), no qual ficam estabelecidos os deveres e responsabilidades dos profissionais brasileiros. De modo geral, o farmacêutico poderá interagir com o profissional prescritor no intuito de garantir segurança no tratamento medicamentoso, observar o uso racional de medicamentos, prover a consulta farmacêutica, informar e orientar o paciente quanto à posologia, interação medicamentosa, interação com alimentos, reações adversas e conservação do produto. Também fica assegurado ao farmacêutico o direito de decidir, quando justificado, sobre o aviamento ou não de qualquer prescrição (CONSELHO..., 2014).

O Manual de Boas Práticas Farmacêuticas desenvolvido pela Agência Nacional de Vigilância Sanitária também regulamenta a profissão farmacêutica, sendo legalmente determinado que o farmacêutico pode exercer, além das funções de dispensação e informação, Atenção Farmacêutica por meio de protocolos, Atenção Farmacêutica domiciliar, aferição de parâmetros fisiológicos e bioquímicos, administração de medicamentos por meio da inalação, bem como serviço de nebulização e aplicação de injetáveis (ANVISA, 2012).

O comparativo da legislação, de forma não aprofundada, de ambos os países, chama a atenção por serem muito parecidas. Observa-se que o farmacêutico brasileiro encontra amparo na legislação para realizar atenção farmacêutica tal qual o profissional canadense, porém ainda não possui autonomia na decisão sobre as medicações dos pacientes. Apesar de poder prescrever medicamentos que não exijam prescrição médica, o farmacêutico não pode decidir sobre a prescrição sem o consentimento do médico. Por exemplo, o farmacêutico canadense possui autonomia em sua decisão final como profissional, po- dendo substituir medicamentos quando necessário, estender prescrições e ajustá-las quando forem necessárias mudanças na forma farmacêutica, dosagem, quantidade e posologia (CONSELHO..., 2013b; NAPRA, 2009).

Outro ponto importante é o conhecimento dos farmacêuticos brasileiros sobre as suas responsabilidades. Em 2004, Silva e Vieira pesquisaram o conhecimento dos farmacêuticos de uma determinada região sobre legislação sanitária e regulamentação da profissão. Os dados encontrados por eles foram alarmantes: apenas $22 \%$ dos farmacêuticos demonstraram um nível considerado bom sobre a legislação vigente no país e poucos sabiam sobre suas atribuições dentro do estabelecimento (SILVA; VIEIRA, 2004).

O Brasil, no entanto, vem fazendo progressos quanto à formulação de suas leis para a proteção da prática farmacêutica de forma que o farmacêutico tenha mais contato com o paciente. Em 2013 o ConseIho Federal de Farmácia adicionou às atribuições do farmacêutico a realização, quando necessário, da consulta e prescrição farmacêutica de medicações que não exija prescrição médica, realização da anamnese farmacêutica e também avaliação dos resultados de exames clínico-laboratoriais e acesso a informações do paciente (CONSELHO..., 2013a). Em 2016 o ConseIho lançou o ProFar - Programa de Suporte ao Cuidado Farmacêutico na Atenção Básica - que tem como objetivo propagar conhecimento e desenvolver as habilidades necessárias para os farmacêuticos atuarem no "cuidado farmacêutico", conceituado como todo serviço farmacêutico que é prestado centrado no paciente e na promoção à saúde (CONSELHO..., 2016).

\section{Metodologias de atenção farmacêutica}

Quanto às metodologias utilizadas, um bom exemplo na Atenção Farmacêutica está a província de Ontário, no Canadá, que conta com dois principais programas reconhecidos pelo governo para a execução das ações de atenção farmacêutica: o Pharmaceutical Opinion Program e o MedsCheck. O Pharmaceutical Opinion Program é uma intervenção clínica feita pelo farmacêutico durante a dispensação de medicamento ou quando estão sendo realizadas avaliações utilizando o MedsChe$c k$, quando o farmacêutico identifica um potencial alvo para intervenção que requer revisão médica, podendo sugerir a interrupção ou adição de medicamentos em uma prescrição até que ocorra uma nova consulta com o médico. O MedsCheck, é um programa que busca uma revisão da farmacoterapia dos pacientes de forma crítica, é um encontro que ocorre anualmente entre o paciente e o farmacêutico, e tem por objetivo a identifi- 
cação dos Problemas Relacionados com Medicamentos de pacientes que fazem o uso de medicamentos de uso crônico (CANADA, 2015; PAMMETT; JORGENSON, 2014).

No Brasil não existem programas oficiais na área de Atenção Farmacêutica em farmácias comerciais de forma continuada. Os serviços quando prestados são mínimos e ocorrem de forma não padronizada, sem a utilização de uma metodologia ou tecnologia específica. Observa-se que as atividades de Atenção Farmacêutica no Brasil são desenvolvidas em sua maioria na área hospitalar e geralmente por instituições de ensino superior ou órgãos privados e públicos vinculados a elas. Em seu relatório, o Consenso Brasileiro de Atenção Farmacêutica afirmou que apesar do entendimento da importância da Atenção Farmacêutica ainda deve ser explorada a aplicação dessas ações, pois existem dificuldades relacionadas sobre a compreensão das formas de medir os resultados encontrados (AMBIEL; MASTROIANNI, 2013; BRASIL, 2004).

Entre as metodologias citadas na literatura brasileira para a Atenção Farmacêutica encontram-se Método Dáder, o Pharmacotherapy WorkUp e o Therapeutic Outcomes Monitoring (TOM). Entre essas opções de protocolos, o Método Dáder é o mais utilizado e tem como base a avaliação dos problemas relacionados a medicamentos, e suas ações estão direcionadas para a intervenção de interações e problemas farmacoterapêuticos (CORRER; OTUKI, 2011; CONSENSO..., 2002). Atualmente, encontra-se também disponível a série "Cuidados Farmacêuticos na Atenção Básica" para instalação de serviços clínicos farmacêuticos nas Unidades Básicas de Saúde, com projeto-piloto iniciado na cidade de Curitiba-PR (BRASIL, 2016 a, b, c, d).

Apesar de esses métodos serem citados como os mais utilizados na literatura brasileira para a pesquisa da atenção farmacêutica, estudo dirigido por Pereira e Freitas (2008), destaca que no Brasil existem casos isolados de interesse do farmacêutico pela realização de ações de Atenção Farmacêutica em suas drogarias, e que a maioria dos trabalhos é realizada por universidades e docentes. Oliveira et al. (2017) ressaltam que isso se deve ao modelo tradicional, centrado no medicamento, e que os estabelecimentos farmacêuticos precisam se readequar para serem prestadores de assistência e atenção farmacêutica. Araújo et al. (2017) avaliaram as atividades de natureza clínica na atenção básica no Brasil, em que foram entrevistados os responsáveis pela entrega dos medicamentos. Destes, 285 eram farmacêuticos e 206 não realizavam tais atividades. Os principais motivos alegados pelos farmacêuticos para não realizar atividades clínicas foram: nunca ter sido solicitado, não dispor de espaço físico e de tempo suficiente.

\section{Modelos de Educação Superior}

Quanto à educação em Farmácia, em 2014 o Brasil possuía 428 cursos de Farmácia credenciados junto ao Ministério de Educação (UFG, 2015), enquanto o Canadá contava com a presença de 10 Instituições de Ensino no seu território com programas de Graduação em Farmácia no ano de 2008 (AUSTIN; ENSOM, 2008). O farmacêutico graduado em universidades brasileiras recebe o título de Farmacêutico Generalista, pois além de trabalhar diretamente com medicamentos e saúde pública, está apto para trabalhar em diversas áreas, como análises bioquímicas, toxicológicas e alimentares. Observa-se também dificuldades na padronização dos currículos das universidades que oferecem o curso de Farmácia devido à falta de infraestrutura, bibliotecas, farmácias-escola, hospitais-escola, laboratórios-escola e outros equipamentos sugeridos pelas Diretrizes Curriculares Nacionais de Graduação em Farmácia (CONSELHO..., DOURADO; COELHO, 2010).

Os farmacêuticos graduados no Canadá possuem como sua principal área de estudo a prática farmacêutica, uma vez que os cursos de Farmácia estão sempre em comunicação com os outros cursos da área da saúde das universidades. Além de trabalhar diretamente no cuidado da saúde do paciente, o farmacêutico pode optar por trabalhar em hospitais, indústrias farmacêuticas e como pesquisadores na investigação de novos fármacos e genéricos. Assim como as universidades brasileiras, no Canadá as instituições de ensino são livres para elaboração dos currículos acadêmicos, filosofia de ensino e métodos de aprendizado, porém os órgãos regulamentadores esperam que os alunos recém-graduados em Farmácia atinjam os níveis esperados de competência profissional. Ainda, após a Graduação em Farmácia o farmacêutico precisa fazer uma avaliação nacional, o Pharmacy Examining Board of Canada, para poder exercer a profissão (CANADIAN..., 2015; FRANKE; LOUIZOS; AUSTIN, 2014; KEHRER; SCHINDE; MANN, 2010; AUSTIN; ENSOM, 2008).

Além disso, a Association of Faculties of Pharmacy of Canada recomendou que até 2020 todas as faculdades destinadas ao ensino das práticas de Farmácia fizessem a adesão ao Doutorado em farmácia (PharmD), para que no futuro seja um padrão para poder exercer a profissão no país. Com o PharmD os estudantes estariam mais bem preparados para exercer a profissão, eliminariam a falta de confiança, hesitação para assumir responsabilidades e desconforto na 
hora de tomar decisões, pois teriam um conhecimento mais amplo das doenças, mais habilidades de comunicação, experiência clínica/prática e mais aptidão para trabalhar em equipes multiprofissionais (CANADIAN..., 2015; FRANKE; LOUIZOS; AUSTIN, 2014; KEHRER; SCHINDE; MANN; 2010; AUSTIN; ENSOM, 2008).

É fato que o farmacêutico graduado em universidades brasileiras possui um conhecimento mais amplo do que o farmacêutico canadense, o que lhe dá um campo de trabalho mais variado. Existe, contudo, uma preocupação dos órgãos canadenses para que os farmacêuticos graduados tenham competência para exercer sua principal função, que é o conhecimento dos medicamentos (AUSTIN; ENSOM, 2008). Já no Brasil, estudo dirigido por Silva e Vieira (2004) cita a falha de educação na área da Atenção Farmacêutica como uma barreira para o crescimento desta área no país. Dessa forma, observa-se a necessidade de uma educação mais direcionada a esta área, pois grande parte dos farmacêuticos brasileiros são habilitados para trabalhar na indústria farmacêutica ou análises clínicas (OLIVEIRA; FARHAT; FEGADOLLI, 2012).

\section{CONCLUSÃO}

Em suma, a Atenção Farmacêutica vem se destacando entre as políticas de saúde pelo seu impacto direto na sociedade e economia de um país. As ações de orientação ao usuário são importantes para o funcionamento do sistema de saúde, pois coloca como foco de trabalho o cuidado ao paciente. É de extrema importância, portanto, que haja envolvimento em âmbito nacional para garantir que o Brasil receba a Atenção Farmacêutica, por meio de mudanças realísticas, como aprimoramento e construção de metodologias e legislações específicas, que guiem e orientem seus profissionais. Nesse sentido, o modelo de atenção farmacêutica do Canadá é exemplo e serve de inspiração.

Em complemento, o órgão responsável pela profissão deve assegurar que seus futuros profissionais estejam recebendo ensino de qualidade e adequado para que tenham segurança para atuar na sociedade. Por fim, também o farmacêutico deve aceitar o seu papel na sociedade de prevenir e reduzir problemas relacionados a medicamentos para que então tenhamos uma Atenção Farmacêutica modelo para outros países.

\section{REFERÊNCIAS}

AMBIEL, I. S. S.; MASTROIANNI, P. C. Resultados da atenção farmacêutica no Brasil: uma revisão. Revista de Ciências Farmacêuticas Básica e Aplicada, v. 34, n. 4, p. 469-474, 2013.
AMERICAN PHARMACISTS ASSOCIATION. APhA Pharmaceutical Care Guidelines Advisory Committee. Approved by the APhA Board of Trustees, August 1995. Available from: http://www.pharmacist.com/principles-practice-pharmaceutical-care. Cited: 24 Apr. 2015.

AMIR, M. Assessing the acceptability of community pharmacy based pharmaceutical care services in Karachi. Idea Paper, v. 2, n. 4, Article 59, 2011.

ANVISA. Boas práticas farmacêuticas. 2012. Disponível em: http://portal.anvisa.gov.br/wps/wcm/connect/ a33b1000414f8dff9cae9fa8d08ea2d4/GGIMP-GIMEP+-+30-10-2012+-+Boas+Pr\%C3\%A1ticas+Farmac\%C3\%AAuticas+-+2560.pdf?MOD=AJPERES. Acesso em: 24 abr. 2015.

ARAÚJO, A. L. A.; UETA, J. M.; FREITAS, O. Assistência farmacêutica como um modelo tecnológico em atenção primária à saúde. Revista de Ciências Farmacêuticas Básica e Aplicada, v. 26, n. 2, p. 87-92, 2005.

ARAÚJO, P. S. et al. Atividades farmacêuticas de natureza clínica na atenção básica no Brasil. Revista de Saúde Pública, v. 51, n. Supl. 2:6s, p. 1-11, 2017.

AUSTIN, Z.; ENSOM, M. H. H. International Pharmacy Education Supplement Education of Pharmacists in Canada. The American Journal of Pharmaceutical Education, v. 72, n. 6, 2008.

BRASIL. Casa Civil. Lei no 8.080, de 19 de setembro de 1990. Dispõe sobre as condições para a promoção, proteção e recuperação da saúde, a organização e o funcionamento dos serviços correspondentes e dá outras providências. Diário Oficial da União, v. 20, 1990.

BRASIL. Resolução no 338, de 6 de maio de 2004. Aprova a Política Nacional de Assistência Farmacêutica. Diário Oficial da União, 2004.

BRASIL. Ministério da Saúde. Lei no 13.021 de 8 de agosto de 2014. Dispõe sobre o exercício e a fiscalização das atividades farmacêuticas. Diário Oficial da União, 11 de agosto de 2014 - Edição extra.

BRASIL. Ministério da Saúde. Cuidado na Atenção Básica. Caderno 1. Disponível em: http://bvsms.saude.gov.br/bvs/ publicacoes/servicos_farmaceuticos_atencao_basica_saude.pdf. Acesso em: 29 ago. 2016a.

BRASIL. Ministério da Saúde. Cuidado na Atenção Básica. Caderno 2. Disponível em: http://bvsms.saude.gov.br/bvs/ publicacoes/cuidado_farmaceutico_atencao_basica_saude_2.pdf. Acesso em: 29 ago. 2016b.

BRASIL. Ministério da Saúde. Cuidado na Atenção Básica. Caderno 3. Disponível em: http://bvsms.saude.gov.br/bvs/ publicacoes/cuidado_farmaceutico_atencao_basica_saude_3.pdf. Acesso em: 20 ago. 2016c.

BRASIL. Ministério da Saúde. Cuidado na Atenção Básica. Caderno 4. Disponível em: http://bvsms.saude.gov.br/bvs/ publicacoes/resultado_projeto_implantacao_cuidados_farmaceuticos.pdf. Acesso em: 20 ago. 2016d.

CANADA. Ontario Public Drug Programs. Ministry of Health and Long-Term Care. Available from: http://www.health. gov.on.ca/en/pro/programs/drugs/phar maopinion/publications/docs/pharmaopinion_faq.pdf. Cited: 24 abr. 2015.

CANADIAN PHARMACISTS ASSOCIATION. Pharmacists in Canada. Available from: http://www.pharmacists.ca/index.cfm/pharmacy-in-canada/pharmacists-incanada/. Cited: 24 Apr. 2015. 
CONSELHO FEDERAL DE FARMÁCIA. Resolução N. 585 de 29 de agosto de 2013. Regulamenta as atribuições clínicas do farmacêutico e dá outras providências. Brasília: Conselho Federal de Farmácia, 2013a.

CONSELHO FEDERAL DE FARMÁCIA. Resolução N. 596 de 21 de fevereiro de 2014. Dispõe sobre o Código de Ética Farmacêutica, o Código de Processo Ético e estabelece as infrações e as regras de aplicação das sanções disciplinares. Brasília: Conselho Federal de Farmácia, 2014.

CONSELHO FEDERAL DE FARMÁCIA. Resolução N. 586, de 29 de agosto de 2013. Regula a prescrição farmacêutica e dá outras providências. Brasília: Conselho Federal de Farmácia, 2013b.

CONSELHO FEDERAL DE FARMÁCIA. Farmácias: uma abordagem sanitária. Disponível em: http://www.cff.org.br/noticia.php?id=1628. Acesso em: 24 abr. 2015.

CONSELHO FEDERAL DE FARMÁCIA. Programa de Suporte ao Cuidado Farmacêutico na Atenção à Saúde - Profar. Conselho Federal de Farmácia. Brasília: Conselho Federal de Farmácia, 2016. 76 p.: il.

CONSENSO BRASILEIRO DE ATENÇÃO FARMACÊUTICA. Atenção Farmacêutica no Brasil: "Trilhando Caminhos". Brasília: Organização Pan-Americana da Saúde, 2002. 24p.

CORRER, C. J.; OTUKI, M. F. Método clínico de atenção farmacêutica. Disponível em: http://www.saude.sp.gov.br/resources/ipgg/assistencia-farmaceutica/otuki-metodoclinicoparaatencaofarmaceutica.pdf. 2011. Acesso em: 24 set. 2015.

DOURADO, S. M. E.; COELHO, M. S. R. Adequação dos cursos de Farmácia às novas Diretrizes Curriculares. Revista Científica da FSA, v. 7, n. 1, p. 129-141, 2010.

FARINA, S. S.; ROMANO-LIEBER, N. S. Atenção farmacêutica em farmácias e drogarias: existe um processo de mudança? Saúde e Sociedade, São Paulo, v. 18, n. 1, p. 7-18, 2009.

FARRIS, K. B; FERNANDO FERNANDEZ-LLIMOS, F.; BENRIMOJ, S. I. Pharmaceutical Care in Community Pharmacies: Practice and Research from Around the World. The Annals of Pharmacotherapy, v. 39, p. 1.539-1.541, 2005.

FIOCRUZ. Histórico da saúde pública. Disponível em: http:// www.ensp.fiocruz.br/portal-ensp/historico-da-saude/. Acesso em: 24 abr. 2015.

FILHO, J. R.; BATISTA, L. M. Perfil da atenção farmacêutica nas farmácias comerciais no município de João Pessoa-PB. Revista Brasileira de Farmácia, v. 92, n. 3, p. 137-141, 2011. FRANKE, G.; LOUIZOS, C.; AUSTIN, Z. Canadian Educational Approaches for the Advancement of Pharmacy Practice. The American Journal of Pharmaceutical Education, v. 78, n. 7, p. 1-9, 2014.

HEPLER, C. D. The third wave in pharmaceutical education: the clinical movement. American Journal of Pharmaceutical Education, v. 51, n. 4, p. 369-385, 1987.

HEPLER, C. D.; STRAND, L. M. Opportunities and responsibilities in pharmaceutical care. American Journal of Hospital Pharmacy, v. 47, n. 3, p. 533-543, 1990.

KEHRER, J. P.; SCHINDE, T. J.; MANN, H. J. Cooperation in Pharmacy Education in Canada and the United States. The American Journal of Pharmaceutical Education, v. 74, n. 8, p. 1-6, 2010.
MARCHILDON, G. P. Canada: Health system review. Health Syst Transit., v. 15, n. 1, p. 1-179, 2013.

MORAK, S.; VOGLER, S.; WALSER, S.; KIJLSTRA, N. Understanding the Pharmaceutical Care Concept and applying it in Practice. Austrian Federal Ministry of Health. Vienna, May 2010. Available from: http://whocc.goeg.at/Literaturliste/ Dokumente/BooksReports/Gesamt\%20Publikation\%20 Understanding\%20the\%20Pharmaceutical\%20Care\%20 Concept\%20and\%20Applying\%20it\%20in\%20Practice.pdf. Cited: 24 abr. 2015.

NAPRA. Model Standards of Practice for Canadian Pharmacists. 2009. Available from: http://napra.ca/Content_Files/ Files/Model_Standards_of_Practice_for_Canadian_Pharmacists_March2009.pdf. Cited: 24 abr. 2015.

NAPRA. Scope of Pharmacy Practice in Canadian Jurisdictions Pan-Canadian Overview. Effective October, 2012. Available from: http://napra.ca/Content_Files/Files/Scope_ of_Practice_Pharmacists_Oct2012.pdf. Cited: 24 abr. 2015.

OLIVEIRA, T. C. A.; FARHAT, F. C. L. G; FEGADOLLI, C. Implantação de protocolo de orientação farmacêutica para indivíduos com Diabetes mellitus em farmácia comunitária. Revista Brasileira de Farmácia, v. 93, n. 3, p. 379-384, 2012.

OLIVEIRA, N. V. B. V. et al. Atuação profissional dos farmacêuticos no Brasil: perfil sociodemográfico e dinâmica de trabalho em farmácias e drogarias privadas. Saúde e Sociedade, v. 26, n. 4, p. 1.105-1.121, 2017.

OPAS. Organização Pan-Americana de Saúde. Consenso Brasileiro de Atenção Farmacêutica: proposta. Brasília, DF, 2002.

OMS. Organização Mundial da Saúde. The role of the pharmacist in the health care system. Geneva: OMS, 1994. 24p.

PAMMETT, R.; JORGENSON, D. Eligibility Requirements for Community Pharmacy Medication Review Services in Canada. Revue Des Pharmaciens Du Canada. Canadian Pharmacists Journal, v. 147, n. 1, p. 20-24, 2014.

PEREIRA, L. R. L.; FREITAS, O. A evolução da Atenção Farmacêutica e a perspectiva para o Brasil. Revista Brasileira de Ciências Farmacêuticas, v. 44, n. 4, p. 601-612, 2008.

PNDU. Relatório do Desenvolvimento Humano. A ascensão do Sul: progresso humano num mundo diversificado, 2013. Disponível em: http://www.pnud.org.br/hdr/arquivos/ RDHglobais/hdr2013_portuguese.pdf. Acesso em: 24 abr. 2015.

SILVA, L. R.; VIEIRA, E. M. Conhecimento dos farmacêuticos sobre legislação sanitária e regulamentação da profissão. Revista de Saúde Pública, v. 38, n. 3, p. 429-437, 2004.

SOUZA, Renilson Rehen. O Sistema Público de Saúde Brasileiro. SEMINÁRIO INTERNACIONAL - TENDÊNCIAS E DESAFIOS DOS SISTEMAS DE SAÚDE NAS AMÉRICAS, 11., 2002, São Paulo, 2002.

UFG. Universidade Federal de Goiás. Número de cursos de Farmácia cresce 343\% na última década, mas mercado de trabalho ainda tem áreas em expansão. Disponível em: https:// www.farmacia.ufg.br/n/33284-numero-de-cursos-de-farmacia-cresce-343-na-ultima-decada-mas-mercado-de-trabalho-ainda-tem-areas-em-expansao. Acesso em: 25 ago. 2015. 